\title{
Efficiency Enhancement of Scattering Near-Field Probes
}

\author{
Tom Siday and Oleg Mitrofanov \\ Electronic and Electrical Engineering, University College London, London, WC1E 7JE, UK
}

\begin{abstract}
We measure, for the first time, the scattering efficiency of resonant terahertz (THz) probes for scattering-type $\mathrm{THz}$ nearfield microscopy. We fabricate the probes by placing an indium 'antenna' directly on the tine of a quartz tuning fork (QTF), which we use as an atomic force microscope (AFM) probe in tapping mode. THz time-domain spectroscopy (TDS) of the THz field scattered from the probe shows that the scattering efficiency of the indium antenna exhibits resonant enhancement determined by the antenna length. These resonant scattering probes can enable $\mathrm{THz}$ near-field imaging applications where THz contrast is weak, such as $2 \mathrm{D}$ materials or biological systems.
\end{abstract}

\section{INTRODUCTION}

$\mathrm{S}$ CATTERING-TYPE near-field microscopy (s-SNOM) at $\mathrm{THz}$ frequencies has been demonstrated with spatial resolution as high as a few nanometers [1]. However, this high spatial resolution comes at a cost: low detection sensitivity, meaning many $\mathrm{THz}$ systems simply cannot be observed with the scattering probe technique.

Low detector sensitivity limits many $\mathrm{THz}$ techniques, and whilst this remains true for scattering probes, in recent years the problem has been mitigated to some extent by using specialized detection schemes such as self-mixing in quantum cascade lasers (QCLs) [2]. However, a more fundamental issue limits the technique's sensitivity: inefficient scattering of the nearfield interaction at the probe apex into the far-field.

Recent studies have shown that designing probes to act as resonant dipole antennas may mitigate the problem of low scattering efficiency, however these studies measured the field at the probe apex[3], [4]. Whilst this provides an indication of the scattering efficiency, it is not a direct measure.

Here, we directly measure the scattering efficiency of $\mathrm{THz}$ near-field probes, which are fabricated from indium directly to the tine of a quartz tuning fork (QTF). Figure 1 shows scanning electron micrographs (SEMs) of several indium probes. The QTF provides sensitive probe-sample distance control when combined with AFM feedback electronics.

\section{Results}

To directly measure the scattering efficiency of the indium tips, the QTF is integrated into a THz time-domain spectroscopy (TDS) system. The AFM feedback electronics are used to tap the indium tip nanometers above a metal surface, and by demodulating the TDS signal at the tapping frequency of the QTF $(\sim 32 \mathrm{kHz})$, we detect fields originating primarily from the tip apex. Figure 2(a) shows the THz field scattered by the indium tips shown in Figure 1(a). Clearly, the THz response of each tip is different.

To better understand the origin of the measured $\mathrm{THz}$ response, we determine the scattering efficiency of the indium tips as a function of frequency. The spectrum of the field scattered by the indium tip $\left(E_{t i p}\right)$ is normalized to spectrum of the THz pulse incident on the tip $\left(E_{i n c}\right)$. Figure 2(b) shows this ratio for each indium tip. These plots of scattering efficiency
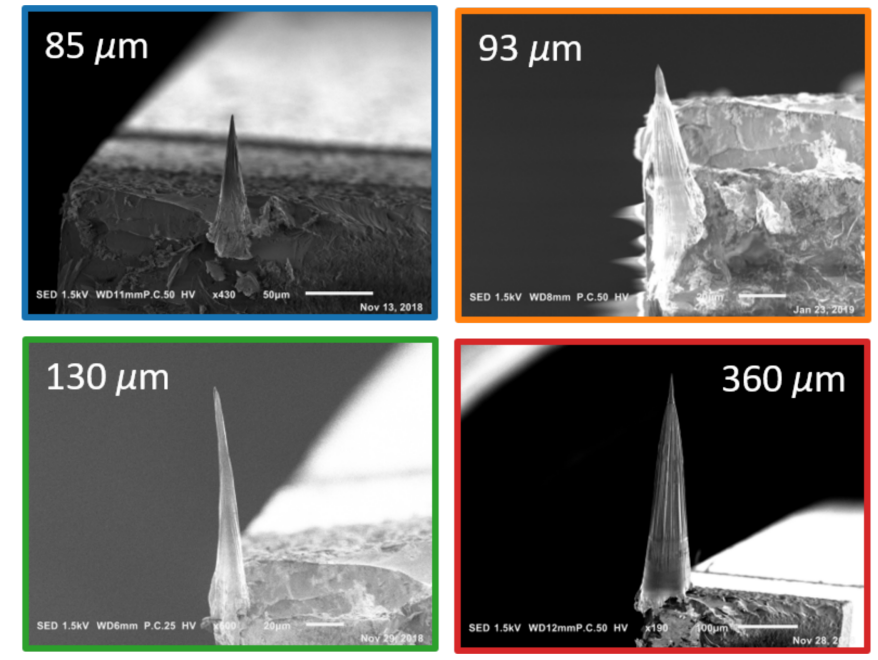

Fig. 1. (a) Scanning electron micrographs (SEM) of Indium tips of varying lengths. The border color of each SEM image in (a) corresponds the curves of the same color in Figure 2 (a) and (b).

exhibit several effects related to the scattering process. Firstly, we attribute the variation in the amplitude of the scattering peak to variation in the dimensions of the tip apex [Rainer new paper]. What is more important for this study are the peaks in the spectrum, which occur at XX THz for the $85 \mu \mathrm{m}$ long tip, $\mathrm{XX} \mathrm{THz}$ for the $93 \mu \mathrm{m}$ long tip, XX THz for the $130 \mu \mathrm{m}$ long tip and XX THz for the 360 um long tip. We explain the presence of these spectral peaks by comparing our results to the ideal half wave dipole model. An ideal half-wave dipole will be resonant (and therefore scatter efficiently) when its length is half that of the incident wavelength. We therefore expect that if the indium tips are behaving in a similar way to the half wave dipole, the wavelength of the resonant peak will depend linearly on the length of the indium tip. This linear dependence can be seen in the inset of Figure 2 (b), which shows the peak frequency of each resonance as a function of inverse tip length. However, whilst this dependence is linear, the resonant wavelength is not twice the tip length as would be in the case of the ideal dipole. This is due to both environmental factors (the high refractive index of the quartz tuning fork) and the geometry of the indium tips; the conical shape of which broadens and red-shifts the resonant frequency of the tip [3].

\section{SUMMARY}

We have, for the first time, characterized the scattering efficiency of resonant scattering probes, by combining scattering probe microscopy with time-domain spectroscopy. These resonant indium probes provide a significant boost to the sensitivity of the scattering probe technique, and by varying the length of the indium tip we can tune the frequency of the tip resonance. The increased sensitivity provided by these probes provides a route towards imaging samples that so far proven 
difficult at terahertz frequencies, such as topological insulators, 2-dimensional materials and biological systems.
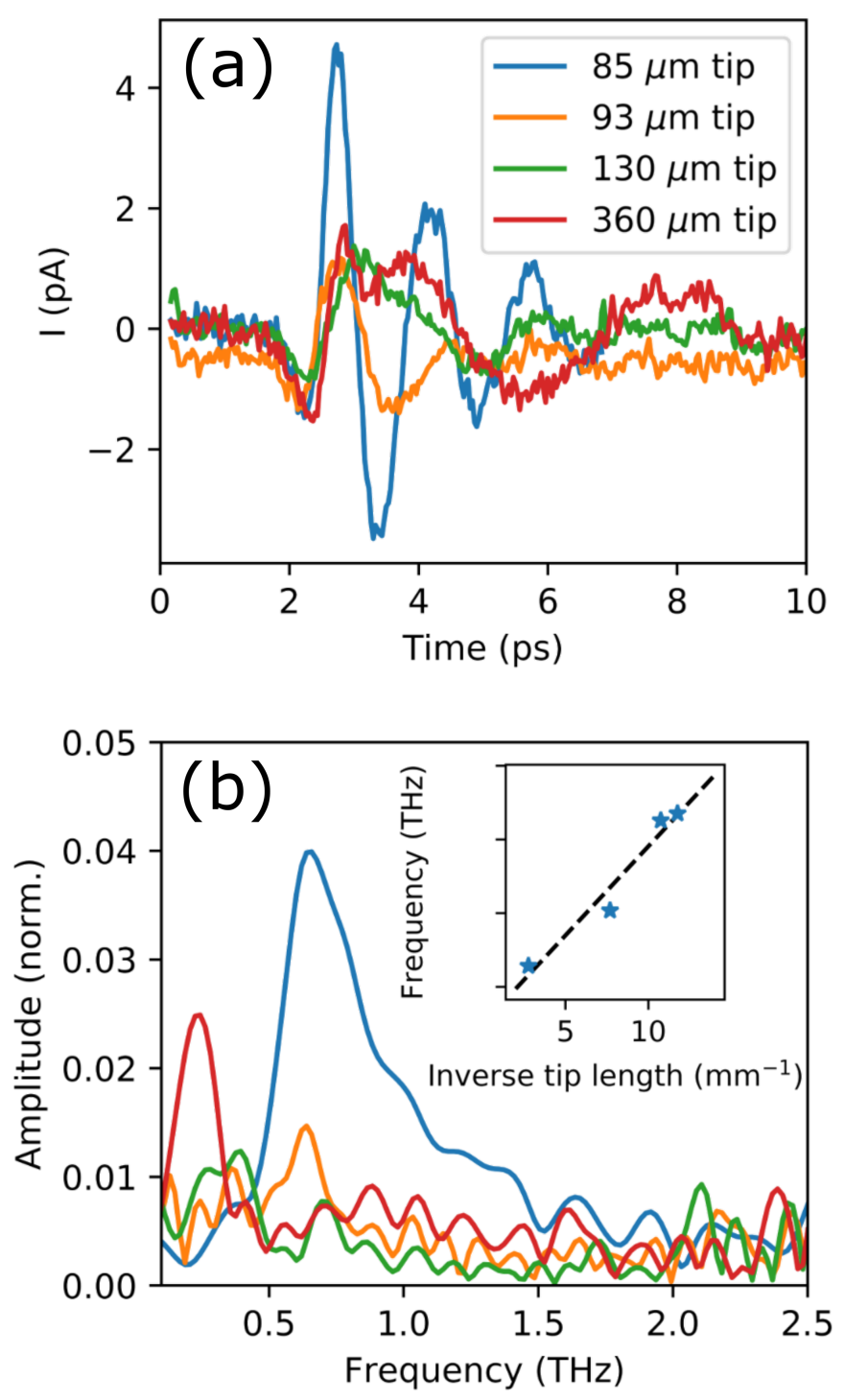

Fig. 2. (a) THz field scattered by the indium tip, measured in the time-domain. (b) Spectral response of the indium tip normalized to the incident field. Inset: frequency of peak field enhancement as a function of inverse tip length.

\section{REFERENCES}

[1] S. Mastel, A. A. Govyadinov, C. Maissen, A. Chuvilin, A. Berger, and R Hillenbrand, "Understanding the Image Contrast of Material Boundaries in IR Nanoscopy Reaching $5 \mathrm{~nm}$ Spatial Resolution," ACS Photonics, vol. 5, no 8, pp. 3372-3378 Jul. 2018.

[2] P. Dean et al., "Apertureless near-field terahertz imaging using the selfmixing effect in a quantum cascade laser," Appl. Phys. Lett., vol. 108, no. 9, p. 091113, Feb. 2016.

[3] T. Siday, M. Natrella, J. Wu, H. Liu, and O. Mitrofanov, "Resonant

terahertz probes for near-field scattering microscopy," Opt. Express, vol. 25, no. 22, p. 27874 , Oct. 2017.

[4] S. Mastel et al., "Terahertz Nanofocusing with Cantilevered TerahertzResonant Antenna Tips," Nano Lett., vol. 17, no. 11, pp. 6526-6533, Nov. 2017. 